Andreas Ledl*

\title{
Evidence-based Human and Social Sciences: Forschungsunterstützung durch systematische Literaturrecherche in den Human- und Sozialwissenschaften
}

https://doi.org/10.1515/bfp-2020-0117

Zusammenfassung: Dieser Artikel möchte für die wachsende Bedeutung von systematischen Übersichtsarbeiten in den Human- und Sozialwissenschaften und die diversen Möglichkeiten der Unterstützung solcher Studien von bibliothekarischer Seite sensibilisieren. Er schildert die Entwicklung systematischer Übersichtsarbeiten in den letzten zehn Jahren, analysiert, welche Rollen dabei Fachreferent*innen bzw. Information Specialists zufallen können und gibt Empfehlungen, welchen konkreten Beitrag die Berufsgruppe der wissenschaftlichen Bibliothekar`innen für solche Forschungsprojekte leisten kann.

Schlüsselwörter: Systematic Review; Meta-Analyse; Humanwissenschaften; Sozialwissenschaften; Forschungsunterstützung

\section{Evidence-based Human and Social Sciences: Research Support through Systematic Literature Research in the Human and Social Sciences}

\begin{abstract}
This article aims to raise awareness of the growing importance of systematic reviews in the human and social sciences and the various ways in which libraries can support such studies. It describes the development of Systematic Reviews over the last ten years, analyses which roles can be played by subject librarians and information specialists and gives recommendations on the concrete contribution that the professional group of academic librarians can make to such research projects.
\end{abstract}

Keywords: Systematic review; meta-analysis; human science; social science; research support
Inhalt

1 Einleitung................... 340

2 Evidence-based Human and Social Sciences

(EBHSS) . . . . . . . . . . . . . . . . . . . . 341

3 Welche Rolle kommt wissenschaftlichen Biblio-

thekar*innen bei der Unterstützung von systema-

tischen Übersichtsarbeiten zu? . . . . . . . . . . . . 345

4 Bei welchen Schritten einer systematischen Übersichtsarbeit sollten wissenschaftliche Biblio-

thekar*innen konkret helfen (können)? . . . . . 347

5 Fazit...................... 348

\section{Einleitung}

Forschungsunterstützung (research support) ist eines der neuen Buzzwords im wissenschaftlichen Bibliothekswesen. Darunter werden Dienstleistungen $\mathrm{zu}$ Themen wie Wissenschaftskommunikation, Open Access, Open Science, Bibliometrie, Informations- und Forschungskompetenz, Forschungsdatenmanagement, wissenschaftliche Integrität, Multimedia, wissenschaftliche Kooperation und nicht zuletzt auch Systematic Reviews subsumiert. ${ }^{1} 2009$ prognostizierten Petra Hätscher und Steffen Wawra, in zehn Jahren werde „aktive Forschungsunterstützung [...] Routine sein". ${ }^{2}$ Nach Ablauf der angesetzten Dekade kann man konstatieren, dass sich diese These in vielen Bereichen bewahrheitet hat. Institutionelle Repositorien gehören an Universitätsbibliotheken mittlerweile ebenso zur Standardausstattung wie Angebote zum Forschungsdatenmanagement. Ganz zu schweigen von der im HypeCycle auf dem produktiven Plateau angekommenen Vermittlung von Informationskompetenz, deren innovativer Stern langsam verblasst - heute ist Digital Literacy angesagt. Hinsichtlich synthetisierender Forschungsmethoden

1 Vgl. Proudman (2015).

2 Hätscher (2009) 70. 
stellt sich die Lage allerdings weit weniger selbstverständlich dar, jedenfalls wenn man von der (Bio-)Medizin und den Gesundheitswissenschaften absieht. Dort wurde z.B. die Dienstleistung „Systematische Literaturrecherche“ der Bibliothek der Medizinischen Fakultät Mannheim schon 2013 als Leuchtturmprojekt beschrieben, da man „seit einigen Jahren einen erhöhten Bedarf an umfassender Beratung $\mathrm{zu}$ komplexen Literaturrecherchen ${ }^{\text {“3 }}$ feststellte. Dieser Bedarf ist mittlerweile auch in den Human- und Sozialwissenschaften spürbar angekommen. Allerdings fällt die Reaktion der wissenschaftlichen Bibliotheken darauf deutlich zaghafter aus als in der evidenzbasierten Medizin (EBM), wo sich inzwischen ganze Teams von „Information Specialists“ um nichts anderes mehr kümmern und aufgrund der steigenden Anforderungen sogar fachspezifische, zertifizierte Aus- und Weiterbildungskurse für das Bibliothekspersonal gefordert werden. ${ }^{4}$

\section{Evidence-based Human and Social Sciences (EBHSS)}

Ein Indiz für die wachsende Bedeutung von Systematic Reviews ${ }^{5}$ in den Human- und Sozialwissenschaften ${ }^{6}$ und damit auch die Notwendigkeit von Forschungsunterstützung in den entsprechenden Fächern ist der 2019 an der Fakultät für Psychologie und Erziehungswissenschaften der KU Leuven (Belgien) veranstaltete, dreitägige, internationale Workshop „Systematic Reviews of Quantitative and/or Qualitative Evidence“. ${ }^{7}$ Bezeichnenderweise wurde er von der „Methodology of Educational Sciences Research Group“ sowie der „Social Research Methodology Group“ in Verbindung mit der „Cochrane Qualitative Research and Implementation Methods Group“ organisiert. Einerseits zeigt sich dadurch, dass systematische Übersichtsarbeiten längst „keine exklusive Methode der EBM mehr“ sind,

3 Metzendorf (2013) 1

4 Vgl. Bissels (2019).

5 „Systematic Review“ wird in diesem Artikel als Sammelbegriff für alle Arten systematischer Übersichtsarbeiten verwendet.

6 Vgl. Gore und Jones (2015) 2, Kocher und Riegelman (2018) 248. Der Fakultäts- bzw. Departementsstruktur der Universität Bern folgend werden in diesem Artikel unter Humanwissenschaften die Fächer Erziehungswissenschaft, Psychologie und Sportwissenschaft, unter Sozialwissenschaften die Fächer Soziologie, Kommunikationsund Medienwissenschaft sowie Politikwissenschaft verstanden. Vgl. https://www.philhum.unibe.ch/forschung/index_ger.html und https://www.wiso.unibe.ch/index_ger.html.

7 https://ppw.kuleuven.be/mesrg/seminars-and-events/sr2019. andererseits - durch die Beteiligung von Cochrane -, dass „das Thema jedoch nach wie vor vom Paradigma der $\mathrm{EBM}^{\star 8}$ dominiert wird.

Systematische Übersichtsarbeiten, von denen es neben den bekannteren Systematic Reviews und Meta-Analysen noch viele weitere Typen ${ }^{9}$ gibt, unterscheiden sich vom klassischen Literature oder Narrative Review durch ihr transparentes und reproduzierbares methodisches Vorgehen, das u. a. beabsichtigt, möglichst alle relevanten Evidenzen zu einer Fragestellung zu finden und in die Analyse und Beantwortung einzubeziehen. Dieser Anspruch auf Vollständigkeit bei der Identifikation von Primärstudien erfordert vielfältige Suchinstrumente und komplexe Suchstrategien.

Auch das Publikationsaufkommen von Studien des Typs Systematic Review stieg in den letzten zehn Jahren kontinuierlich an, weil es angesichts des immensen Forschungsoutputs immer schwieriger wurde, den Überblick $\mathrm{zu}$ behalten oder verlässliche Aussagen zu treffen. Die Formel lautet also, dass sich mit der ,Zunahme an empirischen Studien [...] auch der Bedarf an [...] Meta-Analysen"10 erhöht. Das Deutsche Internationale Institut für Pädagogische Forschung (DIPF) kann exemplarisch für den aktuellen Trend in den Human- und Sozialwissenschaften stehen, Akteuren in herausgehobenen Positionen (hier im Bildungswesen) evidenzbasierte Entscheidungshilfen zur Verfügung zu stellen:

\footnotetext{
„Um Verantwortlichen in Bildungspolitik, -administration und -praxis Entscheidungen auf der Grundlage von empirisch erarbeitetem Wissen zu ermöglichen, bereitet das DIPF die Vielfalt der vorliegenden wissenschaftlichen Erkenntnisse für die verschiedenen Entscheidungsträger in Form von Systematic Reviews (systematische Übersichtsarbeiten) anwendungsorientiert auf. Sie zeichnen sich durch hohe Aussagekraft, wissenschaftliche Qualität und Steuerungsrelevanz aus. Entscheidend für ihre Qualität ist, dass alle relevanten Forschungsergebnisse einbezogen, bewertet und wissenschaftlich fundiert strukturiert werden, bevor sie in die Bilanz einfließen." ${ }^{11}$
}

Der wachsende Bedarf an Evidence-based Human and Social Sciences, welche die Wirksamkeit von Interventionen durch die besten verfügbaren wissenschaftlichen Erkenntnisse absichern, Theorien empirisch überprüfen oder Forschungsdesiderate zu Tage fördern sollen, ist auch anhand von Recherchen in für die einzelnen Disziplinen

\footnotetext{
8 Merz (2016) 4.

9 Vgl. Grant und Booth (2009), Leary und Walker (2018), siehe auch z. B. https://guides.temple.edu/c.php?g=78618\&p=3879604.

10 Weiß und Wagner (2008) 251.

11 Schmuck et al. (2019) 15.
} 
Tab. 1: Dokumentation der in den Datenbanken angewandten Suchabfragen

\begin{tabular}{|c|c|c|}
\hline & Datenbank & Suchstring ${ }^{12}$ \\
\hline \multirow{3}{*}{ 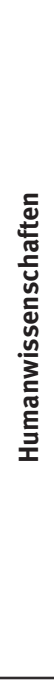 } & $\begin{array}{l}\text { APA PsycINFO (Psychologie) } \\
\text { [Ovid, Advanced Search] }\end{array}$ & 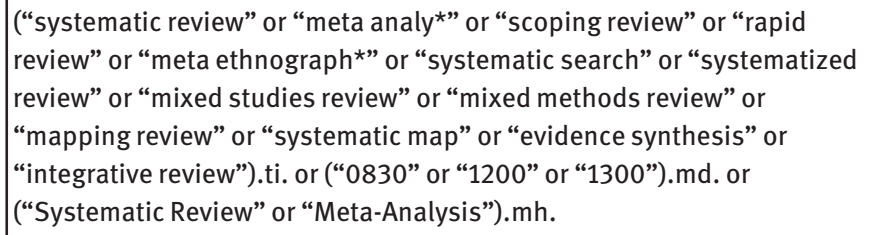 \\
\hline & $\begin{array}{l}\text { ERIC (Erziehungswissenschaft) } \\
\text { [Ovid, Advanced Search] }\end{array}$ & 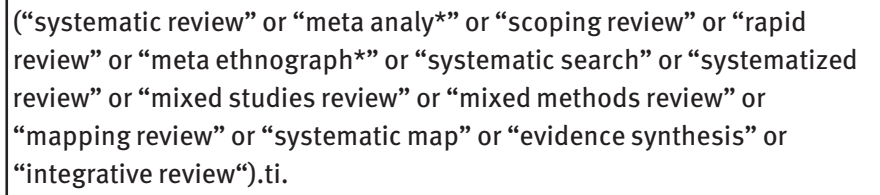 \\
\hline & $\begin{array}{l}\text { SPORTDiscus (Sportwissenschaft) } \\
\text { [EBSCOHost, Advanced Search] }\end{array}$ & 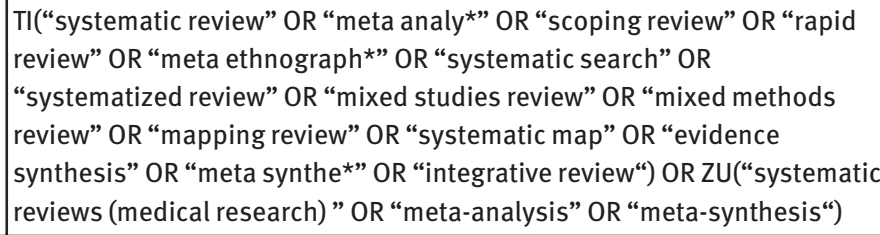 \\
\hline \multirow{4}{*}{ 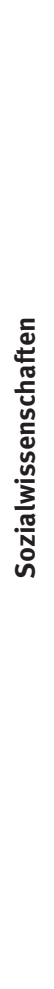 } & $\begin{array}{l}\text { Communication \& Mass Media Complete } \\
\text { (Kommunikations- und Medienwissenschaft) } \\
\text { [EBSCOHost, Advanced Search] }\end{array}$ & $\begin{array}{l}\text { TI(“systematic review” OR “meta analy*” OR “scoping review” OR “rapid } \\
\text { review” OR “meta ethnograph”” OR “systematic search” OR } \\
\text { "systematized review” OR “mixed studies review” OR “mixed methods } \\
\text { review” OR “mapping review” OR “systematic map” OR “evidence } \\
\text { synthesis” OR “meta synthe*” OR “integrative review”) OR ZU } \\
\text { (“systematic reviews (medical research)” OR “meta-analysis" OR “meta- } \\
\text { synthesis”) }\end{array}$ \\
\hline & $\begin{array}{l}\text { Political Science Complete (Politikwissenschaft) } \\
\text { [EBSCOHost, Advanced Search] }\end{array}$ & 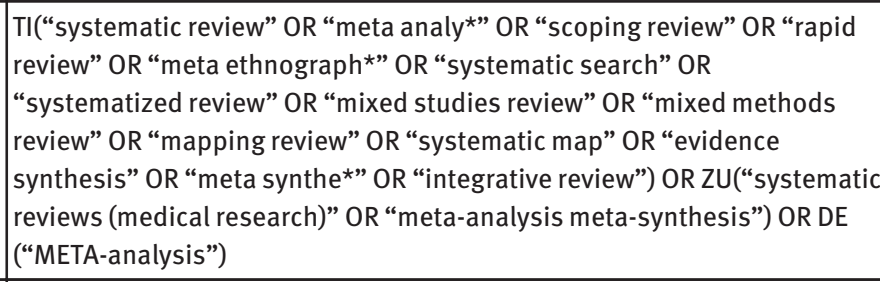 \\
\hline & $\begin{array}{l}\text { SocINDEX (Soziologie) } \\
\text { [EBSCOHost, Advanced Search] }\end{array}$ & 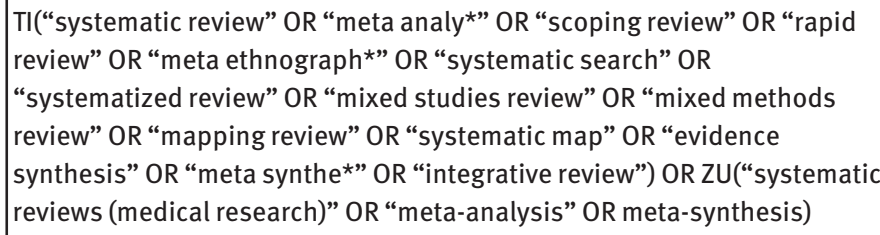 \\
\hline & $\begin{array}{l}\text { Social Sciences Citation Index (SSCI) } \\
\text { [Web of Science Core Collection, Title] }\end{array}$ & $\begin{array}{l}\text { ((“systematic review” OR “meta analy”” OR “scoping review” OR “rapid } \\
\text { review” OR “meta ethnograph*” OR “systematic search” OR } \\
\text { "systematized review” OR “mixed studies review” OR “mixed methods } \\
\text { review” OR “mapping review” OR “systematic map” OR “evidence } \\
\text { synthesis” OR “meta synthe*” OR “integrative review”)) }\end{array}$ \\
\hline$\frac{.5}{\frac{N}{0}}$ & $\begin{array}{l}\text { MEDLINE ALL (Medizin) } \\
\text { [Ovid, Advanced Search] }\end{array}$ & 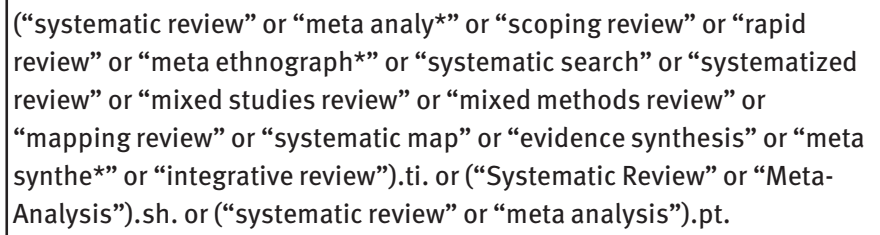 \\
\hline
\end{tabular}

12 Die Suchstrategie kann im ersten Teil auf das Titel-Feld (ohne Abstract und Author Keywords) begrenzt werden, da die relevanten Studien sich in der Regel durch einen Titelzusatz als systematische Übersichtsarbeit zu erkennen geben. Vgl. Sutton et al. (2019) 210. 


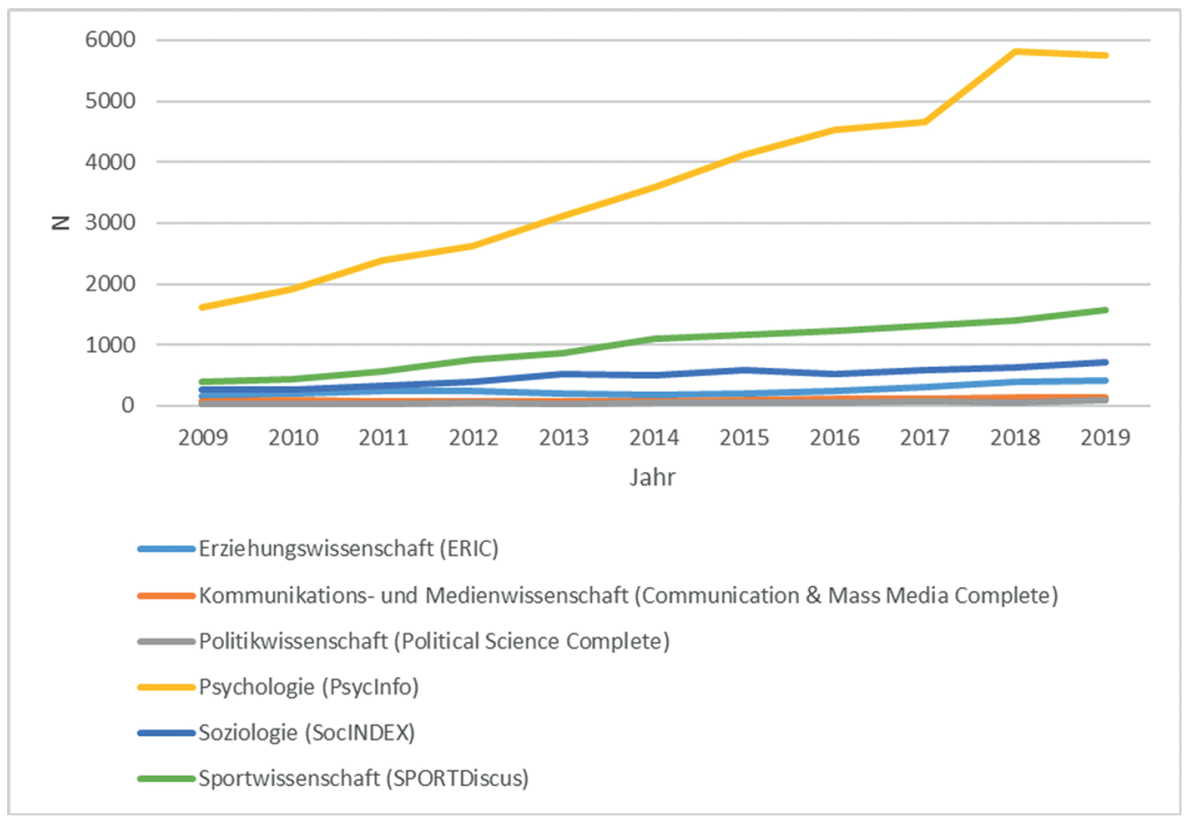

Abb. 1: Systematische Übersichtsarbeiten pro Jahr nach Disziplin und Fachdatenbank (2009-2019)

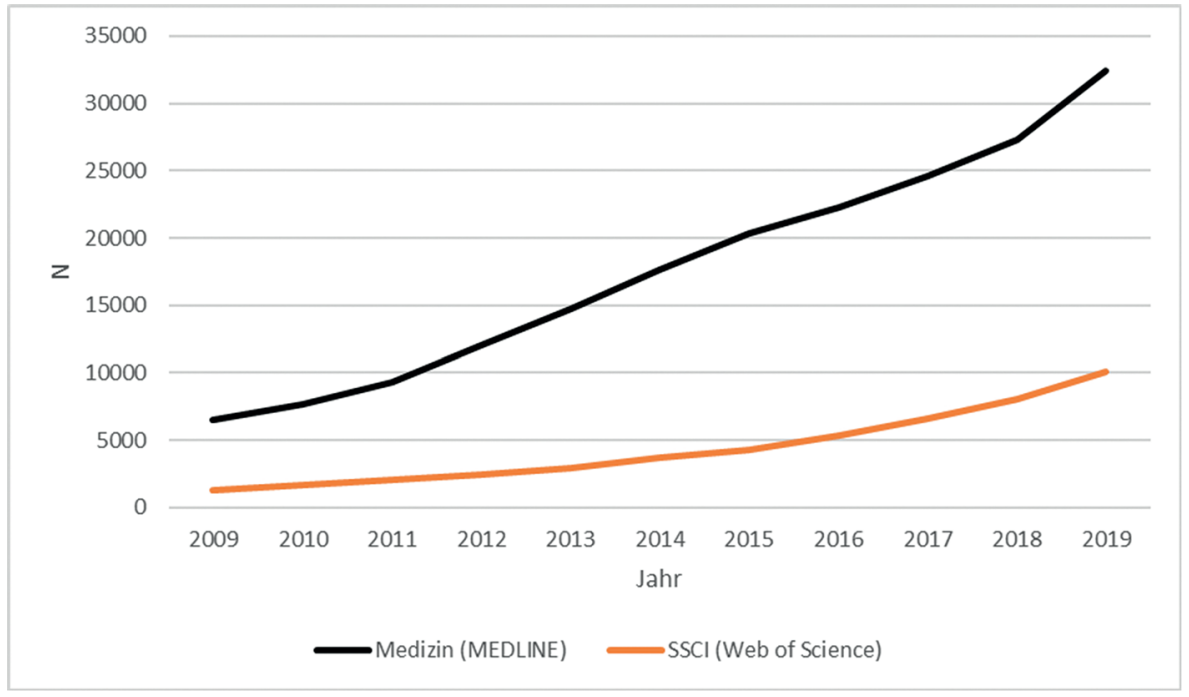

Abb. 2: Systematische Übersichtsarbeiten pro Jahr in MEDLINE und dem SSCI (2009-2019)

repräsentativen, bibliografischen Fachdatenbanken ablesbar. Für den Untersuchungszeitraum 2009-2019 wurden am 24. November 2020 die in Tab. 1 aufgeführten Datenbanken durchsucht.

Vergleicht man punktuell die Werte von 2009 und 2019, haben systematische Übersichtsarbeiten in der Kommunikations- und Medienwissenschaft um den Faktor 1.68, in der Erziehungswissenschaft um den Faktor 2.51, in der Soziologie um den Faktor 2.74, in der Politikwissenschaft um den Faktor 3.38, in der Psychologie um den Faktor 3.56 und in der Sportwissenschaft um den Faktor 4.04 zugenommen. In absoluten Zahlen bedeutet das ei- nen Anstieg von 77 auf 129 Publikationen in der Kommunikations- und Medienwissenschaft, von 163 auf 409 Publikationen in der Erziehungswissenschaft, von 258 auf 708 Publikationen in der Soziologie, von 29 auf 98 Publikationen in der Politikwissenschaft, von 1614 auf 5752 Publikationen in der Psychologie und von 390 auf 1576 Publikationen in der Sportwissenschaft (vgl. Abb. 1). Zu beachten ist, dass die Psychologie 2009 bereits deutlich mehr systematische Übersichtsarbeiten aufweist als alle anderen Fächer zusammen und auch in der Sportwissenschaft der Sockel an Publikationen dieser Art höher ist als in den übrigen Fächern. Beide haben - das dürfte wohl die Erklärung 
Tab. 2: Anteil in Prozent (AT) der Systematischen Übersichtsarbeiten (SÜ) an der Gesamtzahl (GZ) der pro Jahr in den Datenbanken nachgewiesenen Publikationen

\begin{tabular}{|c|c|c|c|c|c|c|}
\hline & 2009 & 2010 & 2011 & 2012 & 2013 & 2014 \\
\hline \multirow[t]{3}{*}{ Psycinfo } & GZ: 163500 & GZ: 166892 & GZ: 175643 & GZ: 185349 & GZ: 191828 & GZ: 195924 \\
\hline & SÜ: 1614 & SÜ: 1922 & SÜ: 2377 & SÜ: 2630 & SÜ: 3110 & SÜ: 3582 \\
\hline & AT: $0.99 \%$ & AT: $1.15 \%$ & AT: $1.35 \%$ & AT: $1.42 \%$ & AT: $1.62 \%$ & AT: $1.83 \%$ \\
\hline Comm. \& Mass & GZ: 31764 & GZ: 28778 & GZ: 28445 & GZ: 27378 & GZ: 25870 & GZ: 25686 \\
\hline \multirow[t]{2}{*}{ Media Complete } & SÜ: 77 & SÜ: 86 & SÜ: 71 & SÜ: 66 & SÜ: 70 & SÜ: 80 \\
\hline & AT: $0.24 \%$ & AT: $0.30 \%$ & AT: $0.25 \%$ & AT: $0.24 \%$ & AT: $0.27 \%$ & AT: $0.31 \%$ \\
\hline \multirow[t]{3}{*}{ ERIC } & GZ: 42598 & GZ: 45932 & GZ: 46230 & GZ: 50551 & GZ: 42749 & GZ: 34304 \\
\hline & SÜ: 163 & SÜ: 189 & SÜ: 239 & SÜ: 232 & SÜ: 204 & SÜ: 171 \\
\hline & AT: $0.38 \%$ & AT: $0.41 \%$ & AT: $0.52 \%$ & AT: $0.46 \%$ & AT: $0.48 \%$ & AT: $0.50 \%$ \\
\hline \multirow[t]{3}{*}{ MEDLINE } & GZ: 785446 & GZ: 824219 & GZ: 877146 & GZ: 942666 & GZ: 997060 & GZ: 1044560 \\
\hline & SÜ: 6505 & SÜ: 7701 & SÜ: 9326 & SÜ: 12037 & SÜ: 14752 & SÜ: 17594 \\
\hline & AT: $0.83 \%$ & AT: $0.93 \%$ & AT: $1.06 \%$ & AT: $1.28 \%$ & AT: $1.48 \%$ & AT: $1.68 \%$ \\
\hline Political Science & GZ: 66801 & GZ: 62357 & GZ: 58408 & GZ: 55009 & GZ: 51330 & GZ: 51843 \\
\hline \multirow[t]{2}{*}{ Complete } & SÜ: 29 & SÜ: 23 & SÜ: 25 & SÜ: 45 & SÜ: 37 & SÜ: 47 \\
\hline & AT: $0.04 \%$ & AT: $0.04 \%$ & AT: $0.04 \%$ & AT: $0.08 \%$ & AT: $0.07 \%$ & AT: $0.09 \%$ \\
\hline \multirow[t]{3}{*}{ SocINDEX } & GZ: 69218 & GZ: 67689 & GZ: 66017 & GZ: 62614 & GZ: 65891 & GZ: 65659 \\
\hline & SÜ: 258 & SÜ: 270 & SÜ: 329 & SÜ: 403 & SÜ: 516 & SÜ: 506 \\
\hline & AT: $0.37 \%$ & AT: $0.40 \%$ & AT: $0.50 \%$ & AT: $0.64 \%$ & AT: $0.78 \%$ & AT: $0.77 \%$ \\
\hline \multirow[t]{3}{*}{ SPORTDiscus } & GZ: 118235 & GZ: 113411 & GZ: 114039 & GZ: 112102 & GZ: 108813 & GZ: 109367 \\
\hline & SÜ: 390 & SÜ: 444 & SÜ: 561 & SÜ: 761 & SÜ: 856 & SÜ: 1104 \\
\hline & AT: $0.33 \%$ & AT: $0.39 \%$ & AT: $0.49 \%$ & AT: $0.68 \%$ & AT: $0.79 \%$ & AT: $1.01 \%$ \\
\hline \multirow[t]{4}{*}{ SSCI } & GZ: 226730 & GZ: 244366 & GZ: 256152 & GZ: 272677 & GZ: 277623 & GZ: 281209 \\
\hline & SÜ: 1330 & SÜ: 1636 & SÜ: 2018 & SÜ: 2496 & SÜ: 2974 & SÜ: 3671 \\
\hline & AT: $0.59 \%$ & AT: $0.67 \%$ & AT: $0.79 \%$ & AT: $0.92 \%$ & AT: $1.07 \%$ & AT: $1.31 \%$ \\
\hline & 2015 & 2016 & 2017 & 2018 & 2019 & \\
\hline \multirow[t]{3}{*}{ PsycINFO } & GZ: 201451 & GZ: 191202 & GZ: 174977 & GZ: 192619 & GZ: 158740 & \\
\hline & SÜ: 4133 & SÜ: 4540 & SÜ: 4659 & SÜ: 5813 & SÜ: 5752 & \\
\hline & AT: $2.05 \%$ & AT: $2.37 \%$ & AT: $2.66 \%$ & AT: $3.02 \%$ & AT: $3.62 \%$ & \\
\hline Comm. \& Mass & GZ: 24098 & GZ: 24530 & GZ: 24910 & GZ: 25017 & GZ: 21347 & \\
\hline \multirow[t]{2}{*}{ Media Complete } & SÜ: 101 & SÜ: 105 & SÜ: 115 & SÜ: 143 & SÜ: 129 & \\
\hline & AT: $0.42 \%$ & AT: $0.43 \%$ & AT: $0.46 \%$ & AT: $0.57 \%$ & AT: $0.60 \%$ & \\
\hline \multirow[t]{3}{*}{ ERIC } & GZ: 35666 & GZ: 40999 & GZ: 42793 & GZ: 42538 & GZ: 37730 & \\
\hline & SÜ: 200 & SÜ: 235 & SÜ: 307 & SÜ: 395 & SÜ: 409 & \\
\hline & AT: $0.56 \%$ & AT: $0.57 \%$ & AT: $0.72 \%$ & AT: $0.93 \%$ & AT: $1.08 \%$ & \\
\hline \multirow[t]{3}{*}{ MEDLINE } & GZ: 1089728 & GZ: 1115001 & GZ: 1125598 & GZ: 1166765 & GZ: 1240018 & \\
\hline & SÜ: 20382 & SÜ: 22232 & SÜ: 24587 & SÜ: 27281 & SÜ: 32441 & \\
\hline & AT: $1.87 \%$ & AT: $1.99 \%$ & AT: $2.18 \%$ & AT: $2.34 \%$ & AT: $2.62 \%$ & \\
\hline Political Science & GZ: 50684 & GZ: 50707 & GZ: 48029 & GZ: 45632 & GZ: 43775 & \\
\hline \multirow[t]{2}{*}{ Complete } & SÜ: 57 & SÜ: 45 & SÜ: 68 & SÜ: 54 & SÜ: 98 & \\
\hline & AT: $0.11 \%$ & AT: $0.09 \%$ & AT: $0.14 \%$ & AT: $0.12 \%$ & AT: $0.22 \%$ & \\
\hline
\end{tabular}

sein - jedenfalls in Teilen Berührungspunkte mit der Medizin, die wie zu erwarten mit einem Faktor von 4.99 bzw. einer Output-Steigerung von 6505 Studien 2009 auf 32441 Studien 2019 den rasantesten Zuwachs erlebte. Führt man die gleiche Suche allerdings im Social Sciences Citation Index (SSCI) von Web of Science durch, ergibt sich mit einem Sprung von 1330 Studien 2009 auf 10101 Studien 2019 relativ sogar ein höherer Zuwachs-Faktor von 7.59 (vgl. Abb. 2).

Auch wenn man die Anzahl an systematisierenden Publikationen jeweils ins Verhältnis zur Gesamtzahl an Publikationen setzt, lässt sich in allen stellvertretend 
durch einzelne Datenbanken untersuchten Disziplinen eine zunehmende Relevanz nachweisen (vgl. Tab. 2). In der Medizin beträgt der Anteil systematischer Übersichtsarbeiten im Vergleich zur in MEDLINE indexierten Jahresproduktion $20192.62 \%$, ausgehend von $0.83 \% 2009$. Insgesamt ist der Output von 2009 (785446) bis 2019 (1240 018) kontinuierlich um den Faktor 1.58 angewachsen, während systematisierende Publikationsformate um den Faktor 4.99 zugenommen haben. In den human- und sozialwissenschaftlichen Datenbanken ist, mit Ausnahme des SSCI, die Gesamtzahl der Publikationen von 2009 bis 2019 jedoch stabil geblieben oder sogar gesunken. Dieser Trend zeigt sich besonders in der Psychologie, wo Systematic Reviews 20193.62 \% aller in PsycINFO nachgewiesenen Dokumente ausmachten, deutlich mehr als in MEDLINE. Gemessen an dem Aufwand, den die evidenzbasierte Medizin für den Aufbau bibliothekarischer Infrastruktur rund um Systematic Reviews seit Jahren betreibt, besteht in der Psychologie wohl der akuteste Handlungsbedarf. Auch im SSCI liegt der Anteil der systematischen Übersichtsarbeiten 2019 mit $2.44 \%$ im Bereich von MEDLINE, gefolgt von SPORTDiscus (2.00\%), SocINDEX (1.15\%), ERIC (1.08\%), Communication \& Mass Media Complete (0.60 \%) und Political Science Complete (0.22 \%). In jeder der herangezogenen Datenbanken wächst die Zahl systematischer Übersichtsarbeiten stärker (+) als die Gesamtzahl der Publikationen, welche fast überall rückläufig ist (-). In PsycINFO beträgt das Verhältnis $3.56(+)$ zu 0.97 (-), in SPORTDiscus 4.04 (+) zu 0.72 (-), in SocINDEX 2.74 (+) zu $0.89(-)$, in ERIC $2.51(+) \mathrm{zu} 0.89(-)$, in Communication \& Mass Media Complete $1.68(+)$ zu $0.67(-)$, in Political Science Complete $3.38(+)$ zu $0.66(-)$, im SSCI 7.59 (+) zu $1.47(+)$.

Systematische Übersichtsarbeiten als Forschungsmethode in den Human- und Sozialwissenschaften sind also „state-of-the-art“"13 und bedürfen der Unterstützung durch bibliothekarische Dienstleistungen. Von Strukturen des Research Supports, wie sie in der evidenzbasierten Medizin vorherrschen, sind die betroffenen Disziplinen aber noch ein gutes Stück entfernt.

\section{Welche Rolle kommt wissenschaftlichen Bibliothekar*innen bei der Unterstützung von systematischen Übersichtsarbeiten zu?}

Systematic Reviews und verwandte Methoden bieten wissenschaftlichen Bibliothekar*innen eine einmalige Gelegenheit, ihre Expertise in Forschungsgruppen einzubringen. Noch dazu müssen Bibliothekar^innen dafür gar nicht mehr zwingend proaktiv an die Forschenden herantreten, denn die fragen die Dienstleistung mittlerweile ihrerseits verstärkt bei den Bibliotheken nach. ${ }^{14}$ Bereits etablierte Beziehungen zu den Instituten/Fakultäten im Sinne des Liaison- bzw. Embedded-Librarian-Konzepts erleichtern selbstverständlich die Kontaktaufnahme. Im sozialwissenschaftlichen Standardwerk zu Forschungssynthese und Meta-Analysen findet sich z. B. die explizite Aufforderung, die Suchstrategie vorab mit einem ${ }^{\star}$ einer wissenschaftlichen Bibliothekar*in $\mathrm{zu}$ diskutieren, vor allem bezüglich Tipps zu möglichen Rechercheinstrumenten. ${ }^{15}$ Die strategische Ausgangssituation könnte besser nicht sein, denn es gibt einen tatsächlichen Bedarf, ja es gehört in diesem methodischen Genre zur guten wissenschaftlichen Praxis, mit Bibliothekar`innen zusammenzuarbeiten. Eventuell fällt eine systematische Übersichtsarbeit im Peer-Review-Verfahren eines Journals sogar durch, weil kein ${ }^{\star}$ Bibliothekar^in einbezogen wurde. Vermehrt werden Systematic Reviews auch als Masterarbeiten durchgeführt ${ }^{16}$ und die Betreuungspersonen vermitteln ihre Studierenden zur Entwicklung oder Besprechung der Suchstrategie an Bibliothekar*innen.

Für human- und sozialwissenschaftliche Bibliotheken ist es deswegen an der Zeit, einen Teil ihrer Mitarbeitenden zu Information Specialists weiterzubilden oder Personen anzustellen, welche die benötigten Kompetenzen mitbringen. Das jeweilige Vorgehen wird stark von der Einschätzung abhängen, ob es sich bei demder Informati-

14 Vgl. Campbell und Dorgan (2015).

15 Cooper (2017) 96, vgl. auch Petticrew und Roberts (2006) 105.

16 „The importance of systematically reviewing scientific literature has been widely acknowledged in the field of education. It follows that the growth potential for systematic reviews conducted as a master thesis project in educational sciences is considerable." Hannes und Van Den Noortgate (2012) 340.

13 Zarghi und Khorasani (2018) 207. 
onsspezialist`in um ein neues Berufsbild „mit eigenen Ausbildungs- und Studiengängen" 17 , oder eher eine Entwicklungstendenz z.B. innerhalb des Fachreferats handelt. Aufgrund des Tätigkeitsprofils - „Informationsspezialisten sammeln, speichern, organisieren, archivieren, suchen und verteilen gedruckte und digitale Informationen“, einschließlich der kooperativen „Durchführung von wissenschaftlichen Recherchen"18 - soll hier die These vertreten werden, dass Fachreferent^innen potenziell exzellente Qualifikationen mitbringen, um sich in Richtung Information Specialists zu bewegen, nicht zuletzt weil sie schon heute die Schnittstelle zwischen Forschung und Bibliothek bilden. Die Transformation von demder „klassischen“ Fachreferent ${ }^{\star}$ in zum Informationsspezialisten mit dem Schwerpunkt forschungsbezogener Services wurde in den Niederlanden schon vor längerer Zeit vollzogen. Besonderer Wert wurde u.a. darauf gelegt, dass „Kenntnisse der Forschungsprozesse und Kontakte zu den Fachbereichen bereits vorliegen “ ${ }^{19}$ Ob dies unbedingt zur Folge haben muss, künftig nur noch Absolvent`innen der eigenen Hochschule einzustellen, sei dahingestellt. Fest steht aber, dass sich der Wandlungsprozess hin zur Forschungsunterstützung nahtlos in die anhaltenden Entwicklungen „von der Bestands- zur Benutzerorientierung“ ${ }^{20}$ bzw. „from collections to connections“"21 einfügt. Für die Umsetzung der forschungsunterstützenden Maßnahmen kann eine Organisation in Teil- oder Bereichsbibliotheken hilfreich sein, wo sich Fachreferent*innen und Information Specialists als Team betrachten, dessen eine Hälfte erwirbt, erschließt und vermittelt, während die andere sich verstärkt um die wissenschaftlichen Dienstleistungen kümmert. Praktiziert wird das beispielsweise an der Bibliothek Medizin ${ }^{22}$ und der für die human- und sozialwissenschaftlichen Fächer zuständigen Bibliothek von Roll $(B v R)^{23}$ der Universitätsbibliothek Bern. Dass sich die Bezeichnung „Informationsspezialistin“ mit dem beschriebenen, neuen Profil dabei über die Medizin hinaus im deutschsprachigen Bibliothekswesen wohl nicht durchsetzen wird, liegt u. a. daran, dass z.B. in der Schweiz schon

17 Cochrane Deutschland Stiftung et al. (2019) 11.

18 Ebd.

19 Schaper (2015).

20 Keller (2012).

21 Tappenbeck (2015).

22 https://www.unibe.ch/universitaet/dienstleistungen/universitae tsbibliothek/teilbibliotheken/medizin/bibliothek_medizin/index_ge r.html\#pane33601.

$23 \mathrm{https} / /$ www.unibe.ch/universitaet/dienstleistungen/universitae tsbibliothek/teilbibliotheken/human_und_sozialwissenschaftliche_ faecher/bibliothek_vonroll_bvr/index_ger.html. ein Studienabschluss bzw. Beruf gleichen Namens, jedoch mit anderen Voraussetzungen, existiert. ${ }^{24}$

Neben der unmittelbaren Beteiligung in Forschungsgruppen können wissenschaftliche Bibliothekar^innen ihr Knowhow auch mittels Schulungen oder Workshops auf Master-Stufe, in Graduate Schools oder Transferable-SkillsProgramme einbringen. Vielerorts gibt es bereits Kurse zum Thema Systematic Reviews bzw. Meta-Analysen, doch diese nehmen hauptsächlich die Frage der Synthetisierung von heterogenen Daten in den Blick. Bezüglich der systematischen Literaturrecherche bleibt es häufig bei dem Hinweis, eine ${ }^{\star} \mathrm{n}$ Informationsspezialist ${ }^{\star}$ in $\mathrm{zu}$ konsultieren. Durch Kooperationen mit Lehrenden aus Instituten oder Fakultäten können wissenschaftliche Bibliothekar`innen diesen fehlenden Baustein ergänzen, wie es z.B. in dem zweitätigen Workshop „How to conduct a systematic review in psychology“25 an der Fakultät für Psychologie der Universität Basel der Fall ist, der von einem PostDoc der Abteilung Klinische Psychologie und Psychotherapie und dem Fachreferat Psychologie gemeinsam unterrichtet wird. Auch eine Zusammenarbeit mit Medizinbibliotheken kann lohnend sein, um interdisziplinäre Angebote aufzubauen, wie z. B. die Veranstaltung „Systematic searching methods for your publication: a researcher's course“ ${ }^{26}$ welche von einer Informationsspezialistin aus der Medizin und dem Fachreferenten für Psychologie an der Universität Bern durchgeführt wird und sich gleichermaßen an (Post)Docs aus der Biomedizin und den Human- und Sozialwissenschaften richtet.

Interessanterweise hat sich das Phänomen, was einst als ein Indikator für den Niedergang des Fachreferats angenommen wurde, nämlich, dass der „Wert einer vernünftigen Recherche ${ }^{\text {“27 }}$ von vielen nicht mehr erkannt werde, ins glatte Gegenteil verkehrt. Gegenwärtig herrscht Konsens darüber, dass die Qualität der Suchstrategie maßgeblichen Einfluss auf die Güte der gesamten Übersichtsarbeit hat, ${ }^{28}$ oder anders ausgedrückt: Komplexe Datenbankrecherchen und solide Rechercheinstrumente sind wieder extrem hip!

24 Vgl. https://www.berufsberatung.ch/dyn/show/1900?id=3323. 25 https://vorlesungsverzeichnis.unibas.ch/de/recherche?id=2418 53.

26 https://www.unibe.ch/research/promotion_of_early_career_rese archers/ts/ts/ressource_veranstaltungen/hs20/systematic_research_ methods/systematic_searching_methods_hs20/index_eng.html\#pan e959884.

27 Schröter (2012) 47.

28 „The search strategy used in systematic reviews is an important consideration, as the comprehensiveness and representativeness of studies identified influences the quality of conclusions derived from the review." Harari et al. (2020) 1. 
Wissenschaftliche Bibliothekar`innen können mit Systematic Reviews aus Workshops, Schulungen, Beratungen und Auftragsrecherchen Einnahmen für ihre Institutionen generieren, die Nutzung des elektronischen Angebots wie Datenbanken, E-Journals und E-Books fördern und selbst publizieren. ${ }^{29}$ Je nachdem wie groß der geleistete Beitrag zur systematischen Übersichtsarbeit war, sollten sie sich auch ermutigt fühlen, eine namentliche Erwähnung in der Publikation oder eine Co-Autorenschaft anzustreben. ${ }^{30}$ Das International Committee of Medical Journal Editors (ICMJE) hat hierfür Kriterien festgelegt, die auch von grossen Teilen der Human- und Sozialwissenschaften übernommen wurden. ${ }^{31}$ Demnach muss als Autor ${ }^{\star}$ in angegeben werden, wer

- wesentlichen Anteil an der Erstellung einer systematischen Übersichtsarbeit bzw. Material dafür beschafft, analysiert oder interpretiert hat,

- zentrale Textpassagen z. B. im Methodenteil selbst verfasst oder überarbeitet hat UND

- die zu publizierende Version genehmigt hat und

- damit auch bereit ist, Verantwortung für alle anderen Teile der Publikation zu übernehmen.

Sind nicht alle vier Voraussetzungen erfüllt, wird zumindest eine Erwähnung empfohlen. ${ }^{32}$

Unabhängig von der konkret erbrachten Unterstützung stehen wissenschaftliche Bibliotheken heute unter einem gewissen Druck, ihren Wert für die Hochschule zu beweisen und Dienstleistungen zu erbringen, die im Einklang mit der institutionellen Ausrichtung stehen. ${ }^{33}$ Das verstärkte Aufkommen systematischer Übersichtsarbeiten in den Human- und Sozialwissenschaften bietet demder wissenschaftlichen Bibliothekar*in die Chance, seine ${ }^{\star}$ ihre Rolle als Partner`in der Forschung wahrzunehmen, sich selbst weiterzubilden, die Unverzichtbarkeit bibliothekarischer Dienstleistungen gegenüber der Hochschulleitung $\mathrm{zu}$ demonstrieren - und so den neuen Erwartungen an wissenschaftliche Bibliotheken gerecht zu werden.
29 Vgl. Gore und Jones (2015) 1.

30 Vgl. Peterson und Rogers (2018).

31 Vgl. Chang (2019) 578f.

32 Vgl. ICMJE (2020).

33 Vgl. Borrego et al. (2018).

\section{Bei welchen Schritten einer systematischen Übersichtsarbeit sollten wissenschaftliche Bibliothekar*innen konkret helfen (können)?}

Forschungsunterstützung durch systematische Literaturrecherche beschränkt sich nicht auf die traditionelle Suche nach Studien allein. Als methodische Anforderung verlangen Systematic Reviews zudem, dass die durchgeführte Recherche transparent und reproduzierbar sein muss. ${ }^{34}$ So ist z.B. das Protokollieren der geplanten Vorgehensweise von Anfang an eine sehr wichtige Aufgabe, um später in der Publikation detailliert über die unternommenen Schritte zu berichten. Das hilft nicht nur Reviewern bzw. Forschenden, das Dargelegte nachzuvollziehen, sondern auch den Autor`innen selbst, um die Studie später, in einem Folgereview, gegebenenfalls zu aktualisieren oder bias zu vermeiden. Für das Reporting steht als Teil des PRISMA Statements das Flow Diagram zur Visualisierung zur Verfügung, für Feedback von bibliothekarischer Seite kann die PRISMA Checklist herangezogen werden. ${ }^{35}$ Das vorab angefertigte Protokoll des Systematic Reviews sollte auf Plattformen wie Open Science Framework $(\mathrm{OSF})^{36}$, Prospero $^{37}$, SocArXiv ${ }^{38}$, PsyArXiv ${ }^{39}$ oder anderen präregistriert werden. Bei der Abfassung des Protokolls assistieren entweder die Eingabemasken der Plattformen, dort werden die benötigten Angaben zu den Grundüberlegungen, der Hypothese und dem methodischen Vorgehen abgefragt. Wo das nicht der Fall ist, liefert die PRISMA-P Checklist wichtige Hinweise..$^{40}$

Das PRISMA-Statement unterteilt den Prozess eines Systematic Reviews in vier Phasen: „Identification“, „Screening“, „Eligibility“ und „Included“. Diese Phasen müssen wissenschaftliche Bibliothekar`innen bzw. Information Specialist kennen und mindestens in die zwei ersten können sie sich qua ihrer ohnehin vorhandenen Kenntnisse einbringen. Die Entwicklung einer passenden Fragestellung für die Literaturrecherche ist ihnen z. B. bereits geläufig, mit PICO, SPIDER und SPICE (und Varianten davon) wurden spezielle Tools zum Formulieren von For-

34 Kocher und Riegelman (2018) 248.

$35 \mathrm{Vgl}$. Moher et al. (2009).

36 Vgl. https://osf.io/.

37 Vgl. https://www.crd.york.ac.uk/prospero/.

38 Vgl. https://osf.io/preprints/socarxiv.

39 Vgl. https://psyarxiv.com/.

40 Moher et al. (2015). 
schungsfragen für systematische, qualitative bzw. quantitative Übersichtsarbeiten entwickelt..$^{41}$ Auch mit den gängigsten Suchoberflächen (Ovid, EBSCO, ProQuest) und Datenbanken (Web of Science, Scopus etc.) kennen sie sich aus, beherrschen deren Syntax (Kommandozeile!) inklusive Field Codes für Title, Abstract, Author Keywords und Subject Headings, und können - auch unter Hinzuziehung von Hilfsmitteln wie etwa der Polyglott Search des Systematic Reviews Accelerators (SRA) ${ }^{42}$ - die Strings entweder kontrollieren (siehe PRESS ${ }^{43}$ ) oder über verschiedene Suchinstrumente hinweg anpassen. Sie wissen zudem, welche kontrollierten Vokabulare in welchen Suchinstrumenten verwendet werden, z. B. MeSH Terms in PubMed, MEDLINE, PsycINFO (neben dem eigenen Thesaurus der American Psychological Association) und Scopus (neben den Emtree Terms). Generell sind sie in der Lage, Empfehlungen zur Datenbankauswahl und Hinweise zu weiteren Literaturquellen für zusätzliche Materialien (Wissenschaftliche Suchmaschinen, Repositorien o. ä.) zu geben. Mittels Suchstrategien wie dem Citation Pearl Growing oder der Umwandlung von Stich- in Schlagwörter können sie die wichtigsten Keywords und Subject Headings für die jeweilige Suchanfrage ermitteln und stimmen diese in einem iterativen Verfahren immer wieder mit den Forschenden ab, bis der ideale String gefunden ist. Die Durchführung der Recherche in den einzelnen Datenbanken gehört ebenso zum Anforderungsprofil wie das Exportieren und Dedublieren der Referenzen in ein resp. mit einem Literaturverwaltungsprogramm (EndNote, Zotero o.ä.). Von dort sollten den Forschenden die bibliografischen Daten so aufbereitet geliefert werden, dass sie sie nur noch in ein Screening Tool (Covidence, Abstrackr, Rayyan uvm. ${ }^{4}$ ) importieren müssen und mit der Auswahl der relevanten Studien beginnen können. Schon nach dem dann folgenden Title/Abstract-Screening sind wissenschaftliche Bibliothekar^innen wieder gefragt, wenn es nämlich darum geht, die Volltexte für die vertiefte, zweite ScreeningPhase zu beschaffen - die nicht immer über Literaturverwaltungsprogramme automatisch importiert werden können, sondern manchmal auch nur mit bibliothekarischem Knowhow zugänglich sind.

Und auch kurz vor Einreichung des Manuskripts stellen sich den Autor*innen häufig Fragen, die Information Specialists beantworten können sollten. Nach dem Screening-Prozess, evtl. der statistischen Auswertung der gefundenen Daten und dem Verfassen des Artikels liegt der

41 Vgl. z. B. https://libguides.murdoch.edu.au/systematic/PICO.

42 Vgl. https://sr-accelerator.com/\#/polyglot.

$43 \mathrm{Vgl}$. McGowan et al. (2016).

44 Vgl. Harrison et al. (2020).
Zeitpunkt der Literaturrecherche meist schon etwas länger zurück. Deshalb ist das Interesse groß, die Suche noch einmal durchzuführen, um möglichst auch die neuesten Artikel mit einzubeziehen. Wie aber kann man die Treffermengen so einschränken, dass wirklich nur Ergebnisse aus dem infrage stehenden Zeitraum ergänzt werden können und die Arbeit nicht wieder von vorne beginnt? Und wie müssen die Suchstrings in der Publikation nachgewiesen werden, damit sie, wie oben erwähnt, transparent und möglichst einfach - am besten per copy \& paste - reproduzierbar sind? Möglicherweise ist auch noch gar nicht klar, bei welchem Journal das Manuskript eingereicht werden kann oder soll, so dass Publikationsberatung erforderlich ist. Und schließlich ergeben sich thematische Anknüpfungspunkte zu Open Access, Bibliometrie und der Verbreitung von wissenschaftlichen Publikationen über die klassischen Kanäle hinaus.

Dieser kurze Abriss mit wenigen Beispielen soll einerseits zeigen, welche vertieften Kenntnisse zur Durchführung systematischer Übersichtsarbeiten notwendig sind, andererseits aber auch verdeutlichen, wie nah die erforderlichen Kompetenzen dem Berufsbild wissenschaftlicher Bibliothekar`innen kommen. MA-Studierende, Doktorierende oder Forschende verfügen meist nicht über die notwendige, detaillierte Erfahrung, um die geschilderten Schritte selbst durchzuführen - oder sie suchen, wie in der einschlägigen Literatur empfohlen - von vornherein den engen Kontakt mit ihren Ansprechpartner*innen in der Bibliothek und integrieren sie als Teil des Forschungsteams in ihr Projekt. Dort übernehmen Bibliothekar^innen dann Funktionen und Aufgaben, die ihnen typischerweise (noch?) nicht zugeschrieben werden.

\section{Fazit}

Es wurde versucht darzulegen, wie wichtig es für humanund sozialwissenschaftliche Fächer bzw. Bibliotheken im deutschsprachigen Raum ist, analog zur Medizin Strukturen der Forschungsunterstützung hinsichtlich systematischer Übersichtsarbeiten aufzubauen. In den USA haben erste Universitätsbibliotheken bereits begonnen, als Antwort auf die wachsende Bedeutung von Systematic Reviews in den empirischen Sozialwissenschaften explizit Dienstleistungen für Forschende aus nichtmedizinischen Disziplinen anzubieten. Unter den „Evidence Synthesis Consultants" der Cornell University Library befindet sich deshalb auch ein „Applied Social Sciences Librarian“ , ${ }^{45}$

45 https://guides.library.cornell.edu/evidence-synthesis/team. 
und die Publikationsliste der Fachreferentin für Sozialwissenschaften der Minnesota University Libraries zeugt jüngst von reger Beschäftigung mit Systematic Reviews und Meta-Analysen. ${ }^{46}$ Mit der Campbell Collaboration ${ }^{47}$ existiert ein internationales sozialwissenschaftliches Forschungsnetzwerk, das ähnlich wie Cochrane systematische Übersichtsarbeiten fördert - jedoch zur evidenzbasierten Beurteilung der Wirksamkeit sozialer Interventionen. Die hauseigenen „Campbell Systematic Reviews“48 sind eine Open-Access-Zeitschrift mit Peer Review, die eine Art methodisch strengen Gold-Standard ${ }^{49}$ gegenüber anderen, als weniger verlässlich erachteten Studien etablieren soll. Auf der Homepage der Campbell Collaboration bzw. dem eigenen YouTube-Kanal ${ }^{50}$ werden außerdem Ressourcen $\mathrm{zu}$ einzelnen Schritten der Durchführung von Systematic Reviews zur Verfügung gestellt. ${ }^{51}$ Für wissenschaftliche Bibliotheken sind die Zeiten sehr günstig, um näher an die Forschung zu rücken und sie durch den Aufbau von Beratungsdienstleistungen im Bereich systematischer Übersichtsarbeiten $\mathrm{zu}$ unterstützen. ${ }^{52}$ Aus eigener Erfahrung kann ich berichten, dass sich der Blick der Fächer, Institute, Fakultäten, Hochschulen und Universitäten auf ihre Bibliotheken und Bibliothekar`innen dadurch völlig verändern wird. Hoffen wir, dass dieser Perspektivwechsel nicht weitere zehn Jahre dauert und sich folgende Einsicht durchsetzt: ,we were lucky enough to have an information specialist who not only attended the systematic review workshop jointly with us, but also played an integral part in setting up the search string “. ${ }^{53}$

\section{Literaturverzeichnis}

Bedenlier, Svenja; Bond, Melissa; Buntins, Katja; Zawacki-Richter, Olaf; Kerres, Michael (2020): Learning by Doing? Reflections on Conducting a Systematic Review in the Field of Educational Technology. Systematic Reviews in Educational Research: Methodology, Perspectives and Application, hg. v. Olaf ZawackiRichter, Michael Kerres, Svenja Bedenlier, Melissa Bond und Katja Buntins, 112-27. Wiesbaden: Springer VS. DOI:10.1007/ 978-3-658-27602-7.

46 https://scholar.google.com/citations?hl=en\&user=u3eP4VYAAA AJ\&view_op=list_works\&sortby=pubdate.

47 Vgl. https://campbellcollaboration.org/.

48 Vgl. https://onlinelibrary.wiley.com/journal/18911803.

$49 \mathrm{Vgl}$. https://campbellcollaboration.org/what-is-a-systematic-revi ew.html.

$50 \mathrm{Vgl}$. https://www.youtube.com/user/collaborationtube/.

51 Vgl. https://campbellcollaboration.org/research-resources/traini ng-courses.html.

52 Siehe umfassend bei Foster und Jewell (2017).

53 Bedenlier et al. (2020) 122.
Bissels, Gerhard (2019): CAS Medical and Health Librarianship: Überlegungen zu einem Spezialstudiengang für das medizinische Informationswesen. Bibliotheksentwicklung im Netzwerk von Menschen, Informationstechnologie und Nachhaltigkeit, hg. v. Simone Fühles-Ubach und Ursula Georgy, 31-37. Bad Honnef: Bock + Herchen.

Borrego, Ángel; Ardanuy, Jordi; Urbano, Cristóbal (2018): Librarians as Research Partners: Their Contribution to the Scholarly Endeavour Beyond Library and Information Science. In: The Journal of Academic Librarianship, 44 (5), 663-70. DOI: 10.1016/j.acalib. 2018.07.012.

Campbell, Sandy; Dorgan, Marlene (2015): What to Do When Everyone Wants You to Collaborate: Managing the Demand for Library Support in Systematic Review Searching. In: Journal of the Canadian Health Libraries Association, 36 (1), 11-19. DOI: 10.29173/ jchla/jabsc.v36i1.24353.

Chang, Yu-Wei (2019): Definition of authorship in social science journals. In: Scientometrics, 118, 563-85. DOI: 10.1007/s11192-0182986-1.

Cochrane Deutschland Stiftung; Institut für Evidenz in der Medizin; Institut für Medizinische Biometrie und Statistik, Freiburg; Arbeitsgemeinschaft der Wissenschaftlichen Medizinischen Fachgesellschaften - Institut für Medizinisches Wissensmanagement; Ärztliches Zentrum für Qualität in der Medizin (2019): Manual Systematische Recherche für Evidenzsynthesen und Leitlinien. 2. Aufl. Verfügbar unter https://freidok.uni-freiburg. de/data/149324.

Cooper, Harris (2017): Research Synthesis and Meta-Analysis. A Stepby-Step Approach. 5. Aufl. Thousand Oaks: Sage (Applied Social Research Methods Series: 2).

Foster, Margaret J.; Jewell, Sarah T. (Hrsg.) (2017): Assembling the Pieces of a Systematic Review: A Guide for Librarians. Lanham, Maryland: Rowman \& Littlefield.

Gore, Genevieve C.; Jones, Julie (2015): Systematic Reviews and Librarians: A Primer for Managers. In: Partnership. The Canadian Journal of Library and Information Practice and Research, 10 (1), 1-16. Verfügbar unter https://journal.lib.uoguelph.ca/index.ph $\mathrm{p} /$ perj/article/view/3343/3505.

Grant, Maria J.; Booth, Andrew (2009): A typology of reviews: an analysis of 14 review types and associated methodologies. In: Health Information \& Libraries Journal, 26 (2), 91-108. DOI: 10.1111/j.1471-1842.2009.00848.x.

Hannes, Karin; Van Den Noortgate, Wim (2012): Master thesis projects in the field of education: who says we need more basic research? In: Educational Research, 3 (4), 340-44.

Harari, Michael B.; Parola, Heather R.; Hartwell, Christopher J.; Riegelman, Amy (2020): Literature searches in systematic reviews and meta-analyses: A review, evaluation, and recommendations. In: Journal of Vocational Behavior, 118, 1-11. DOI: 10.1016/j.jvb.2020.103377.

Harrison, Hannah; Griffin, Simon J.; Kuhn, Isla; Usher-Smith, Juliet A. (2020): Software tools to support title and abstract screening for systematic reviews in healthcare: an evaluation. In: $B M C$ Medical Research Methodology, 20 (7). DOI:10.1186/s12874020-0897-3.

Hätscher, Petra (2009): Wer bewegt das Wissen: Wo stehen wir in zehn Jahren? Wissen bewegen - Bibliotheken in der Informationsgesellschaft. 97. Deutscher Bibliothekartag in Mannheim, hg. v. Ulrich Hohoff, 69-72. Frankfurt am Main: Klostermann. 
ICMJE - International Committee of Medical Journal Editors (2020): Defining the Role of Authors and Contributors. Verfügbar unter http://www.icmje.org/recommendations/browse/roles-andresponsibilities/defining-the-role-of-authors-and-contribu tors.html.

Keller, Alice (2012): „Subject Librarians“ in Großbritannien: Von der Bestands- zur Benutzerorientierung. In: BIBLIOTHEK - Forschung und Praxis, 36 (1), 11-23. DOI:10.1515/bfp-2012-0003.

Kocher, Megan; Riegelman, Amy (2018): Systematic reviews and evidence synthesis: Resources beyond the health sciences. In: College \& Research Libraries News, 79 (5), 248-52. Verfügbar unter https://crln.acrl.org/index.php/crlnews/article/view/169 $67 / 18685$.

Leary, Heather; Walker, Andrew (2018): Meta-analysis and metasynthesis methodologies: Rigorously piecing together research. In: TechTrends, 62 (5), 525-34. DOI:10.1007/s11528-018-0312-7.

McGowan, Jessie; Sampson, Margaret; Salzwedel, Douglas M.; Cogo, Elise; Foerster, Vicki; Lefebvre, Carol (2016): PRESS Peer Review of Electronic Search Strategies: 2015 Guideline Statement. In: Journal of Clinical Epidemiology, 75, 40-46. DOI:10.1016/j.jcli nepi.2016.01.021.

Merz, Anne-Kathrin (2016): Information Behaviour bei der Erstellung systematischer Reviews. Informationsverhalten von Information Professionals bei der Durchführung systematischer Übersichtsarbeiten im Kontext der evidenzbasierten Medizin. Dissertation, Universität Regensburg. Verfügbar unter https://epub.uni-re gensburg.de/36331/1/2017_11_13_akm_final.pdf.

Metzendorf, Maria-Inti (2013): Leuchtturmprojekte an Medizinbibliotheken: „Systematische Literaturrecherche an der Bibliothek der Medizinischen Fakultät Mannheim der Universität Heidelberg“. In: GMS Medizin - Bibliothek - Information, 13 (3):Doc22, DOI:10.3205/mbi000286.

Moher, David; Liberati, Alessandro; Tetzlaff, Jennifer; Altman, Douglas G.; The PRISMA Group (2009): Preferred Reporting Items for Systematic Reviews and Meta-Analyses: The PRISMA Statement. In: Plos Medicine, 6 (7):e1000097, DOI:10.1371/journal. pmed.1000097.

Moher, David; Shamseer, Larissa; Clarke, Mike; Ghersi, Davina; Liberati, Alessandro; Petticrew, Mark; Shekelle, Paul; Stewart, Lesley A.; PRISMA-P Group (2015): Preferred reporting items for systematic review and meta-analysis protocols (PRISMA-P) 2015 statement. In: Systematic Reviews, 4 (1). DOI:10.1186/2046-40 53-4-1.

Peterson, Shenita R.; Rogers, Hannah K. (2018): Credit where credit is due: Authorship among systematic review service librarians. Verfügbar unter https://dc.uthsc.edu/cgi/viewcontent.cgi?arti cle $=1047 \&$ context $=$ scmla.
Petticrew, Mark; Roberts, Helen (2006): Systematic Reviews in the Social Sciences: A Practical Guide. Malden, MA: Blackwell Publishing.

Proudman, Vanessa (2015): Internationale Trends der Forschungsunterstützung in wissenschaftlichen Bibliotheken. Präsentation am Österreichischen Bibliothekartag in Wien, 18. September 2015. Verfügbar unter https://opus4.kobv.de/opus4-bib-info/fil es/2157/OEB_Tag_2015_Final_VProudman.pdf.

Schaper, Christiane (2015): Bibliothek und Forschung - das Beispiel Holland. Verfügbar unter https://aktuelles.uni-frankfurt.de/stu dium/bibliothek-und-forschung-das-beispiel-holland/.

Schmuck-Soldan, Steffen; Klieme, Eckhard; Reh, Sabine (2019): DIPF | Leibniz-Institut für Bildungsforschung und Bildungsinformation. In: Erziehungswissenschaft 30 (59), 9-19. Verfügbar unter http s://www.pedocs.de/volltexte/2019/18191/pdf/EZW_59_2019_ Schmuck-Soldan_Klieme_Reh_DIPF_Leibniz-Institut_Bildungsfo rschung.pdf.

Schröter, Marcus (2012): Fachreferat 2011 - Innenansichten eines komplexen Arbeitsfeldes. In: BIBLIOTHEK - Forschung und Praxis, 36 (1), 32-50. DOI:10.1515/bfp-2012-0005.

Sutton, Anthea; Clowes, Mark; Preston, Louise; Booth, Andrew (2019): Meeting the review family: exploring review types. In: Health Information \& Libraries Journal, 36, 202-22. DOI:10.1111/ hir.12276.

Tappenbeck, Inka (2015): Fachreferat 2020: from collections to connections. In: Bibliotheksdienst, 49 (1), 37-48. DOI:10.1515/ bd-2015-0006.

Weiß, Bernd; Wagner, Michael (2008): Potentiale und Probleme von Meta-Analysen in der Soziologie. In: Sozialer Fortschritt, 57 (10/11), 250-56.

Zarghi, Nazila; Khorasani, Soheil Dastmalchian (2018): EvidenceBased Social Sciences: A New Emerging Field. In: European Journal of Social Science Education and Research, 5 (2), 207-11. DOI:10.2478/ejser-2018-0048.

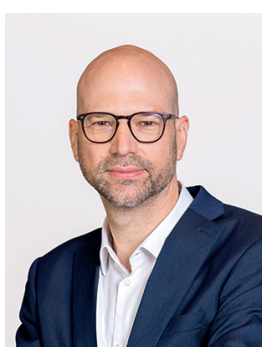

\section{Dr. Andreas Ledl} Fachhochschule Nordwestschweiz (FHNW) Campus Muttenz Bibliothek Hofackerstrasse 30

CH-Muttenz 4132

Schweiz

andreas.ledl1@fhnw.ch 